\title{
Functionalized silica materials for electrocatalysis
}

\author{
VELLAICHAMY GANESAN \\ Department of Chemistry, Faculty of Science, Banaras Hindu University, Varanasi 221 005, India \\ e-mail: velganesh@yahoo.com; velgan@bhu.ac.in
}

MS received 15 March 2014; accepted 17 May 2014

\begin{abstract}
Electrocatalysis is an important phenomenon which is utilized in metal-air batteries, fuel cells, electrochemical sensors, etc. To increase the efficiency of the electrocatalytic process and to increase the electrochemical accessibility of the immobilized electrocatalysts, functionalized and non-functionalized mesoporous organo-silica (MCM41-type-materials) are used in this study. These materials possess several suitable properties to be durable catalysts and/or catalyst supports. Owing to the uniform dispersion of electrocatalysts (metal complex and/or metal nanoparticles (NPs)) on the functionalized and non-functionalized silica, an enormous increase in the redox current is observed. Long range channels of silica materials with pore diameter of 15-100 ̊ allowed metal NPs to accommodate in a specified manner in addition to other catalysts. The usefulness of MCM-41-type silica in increasing the efficiency of electrocatalysisis demonstrated by selecting oxygen, carbon dioxide and nitrite reduction reactions as examples.
\end{abstract}

Keywords. Functionalized silica; electrocatalysis; metal nanoparticles; oxygen reduction; carbon dioxide reduction; nitrite reduction.

\section{Introduction}

Electrodes when modified with silica materials exhibit significant improvements in electrocatalysis and electron transfer kinetics. ${ }^{1-5}$ They combine the intrinsic properties of silica materials to reduction/oxidation of the target analyte leading to the construction of electrochemical sensors and biosensors. ${ }^{1-5}$ Templated routes to synthesize silica materials give rise to wellordered structures with pores and interconnected channels, whereas a non-templated route leads to amorphous silica powder., ${ }^{6,7}$ Both of these (ordered and amorphous) materials can be used as catalysts and/or supports for catalysts. However, considering electron transport and/or charge transport properties on electrode surfaces, it appears beneficial to use ordered mesoporous materials for fast electron and/or charge transfers. ${ }^{1-5,8-10}$ They possess a regular array of uniform nanostructured pores and the pore sizes can be varied over 15 to $100 \AA$ Á through the selection of appropriate template. After the removal of the template, they show large surface area ${ }^{1-7}$ and peculiar adsorption properties, ${ }^{2,11}$ which can lead to potential applications in electrocatalysis. ${ }^{2,11,12}$ Likewise, metal nanoparticles (M NPs) are also equally attractive for utilizing their interesting electronic, optical and catalytic properties, which depend mainly on the size and shape of $\mathrm{M}$ NPs. ${ }^{2,11-23}$ These nano-scaled materials should be suitably immobilized on a substrate to avail their attractive properties for electrocatalytic applications. ${ }^{24} \mathrm{Au}$ and Ag NPs show promising applications in several areas such as fuel cells and electrochemical sensors owing to their striking roles such as catalyst, electrocatalyst, etc. ${ }^{1-5,11-20,22-25}$ One can use silica precursors possessing organic functional groups to stabilize M NPs and to combine the favourable properties of the organic moiety to the inorganic backbone, ${ }^{25}$ which is expected to be attractive in electrocatalytic applications and electrochemical sensing devices. Therefore, for electrocatalytic applications, immobilization of electrocatalyst is necessary as this generally enhances the rate of a reaction and bare electrodes are often not appropriate for these purposes.

In chemically modified electrodes (CMEs), the electrode modifiers are generally allowed to form membrane/film on the surface of the electrodes by dip or spin coating and in most cases, the electrode modifier is a non-conducting material. Earlier in the 1980s when the concept of CMEs were developed, ${ }^{26-29}$ researchers thought that the use of non-conducting material as electrode modifier could be a big obstacle. Though the electron transport properties within the films are supported by hopping mechanism, ${ }^{26-29}$ electrode modifiers generally depress the electron transport properties in CMEs. Depressed electron transport properties can make significant fraction of redox species to be electrochemically silent. Therefore, improving the electron transport properties within the film becomes another 
important direction which is highly desired in electrocatalytic applications. In this context, we decided to anchor $\mathrm{Ag}$ and $\mathrm{Au} \mathrm{NPs}$ inside the channels of thiol functionalized silica materials $s^{2,11,12,18,20}$ in addition to the adsorption/incorporation of an electrocatalyst. When suitable procedures are adopted, metal porphyrins (MPs) and metal phthalocyanines (MPcs) can be conveniently assimilated into these MCM41-type materials. ${ }^{30,31}$ MPs and MPcs are efficient catalysts for various reactions such as oxygen, ${ }^{32,33}$ carbon dioxide, ${ }^{34,35}$ nitrite $e^{36,37}$ and nitrate ${ }^{38,39}$ reductions. In these aspects, cobalt phthalocyanine (CoPc), zinc phthalocyanine $(\mathrm{ZnPc})$ and certain Schiff base complexes are immobilized on thiol functionalized or on a non-functionalized ordered silica and used as electrocatalysts. ${ }^{2,11,18-20,40}$ In this study, it is aimed to demonstrate the successful utilization of MCM-41type silica materials in electrocatalysis. In addition, the effects of Ag and Au NPs on the loaded electrocatalysts towards certain reduction reactions are summarized.

\section{Experimental}

\subsection{Chemicals and reagents}

Triple distilled water was used to prepare aqueous solutions. Analytical grade chemicals were used unless otherwise stated. Silica precursors, mercaptopropyltriethoxysilane $>80 \%$ and tetraethoxysilane $>98 \%$ procured from Sigma-Aldrich were used as received. $\mathrm{ZnPc}, \mathrm{CoPc}, \mathrm{HAuCl}_{4}$ and polystyrene were also procured from Sigma-Aldrich. Cetyltrimethylammonium bromide (CTAB) and $\mathrm{AgNO}_{3}$ was purchased from Himedia and Merck, respectively. Salicylaldehyde, 1,2-phenylenediamine, ethylenediamine and poly(vinyl alcohol) (SD Fine Chemicals Ltd., India) were used without further purification. Following the literature procedures, ${ }^{19,40-42}$ Schiff base ligands salen $\mathrm{H}_{2}$ (N,N-bis(salicylaldehyde) ethylenediamine) and salophen $\mathrm{H}_{2}$ (N,N'-bis(salicylaldehyde)1,2 phenylenediamine) were prepared and purified. $[\mathrm{Co}(\text { salen })]^{+},[\mathrm{Co}(\text { salophen })]^{+},[\mathrm{Mn}(\text { salen })]^{+}$and $[\mathrm{Mn}(\text { salophen })]^{+}$complexes were prepared using literature procedures. ${ }^{19,40-43}$ Template-free thiol functionalized silica and non-functionalized silica materials were synthesized according to the reported one-pot synthetic approach ${ }^{2-5,25}$ and abbreviated as TFS and NFS, respectively. Ag and Au NPs were introduced into the TFS by equilibrating with the corresponding metal ion precursors $\left(\mathrm{AgNO}_{3}\right.$ or $\left.\mathrm{HAuCl}_{4}\right)$ for $12 \mathrm{~h}$ followed by reduction with $\mathrm{NaBH}_{4}$ at $0-4^{\circ} \mathrm{C} .^{2,11,12}$ They were named as Ag-TFS and Au-TFS, respectively. TFS, Ag-TFS and Au-TFS materials were adsorbed with $\mathrm{CoPc}$ or $\mathrm{ZnPc}$ using simple equilibration procedure with the respective molecules in dimethylformamide (DMF). 2,11,18,20 The resulting materials were represented as TFS-ZnPc/CoPc, Ag-TFS-ZnPc/CoPc and $\mathrm{Au}-\mathrm{TFS}-\mathrm{ZnPc} / \mathrm{CoPc}$. Similar equilibration procedures were followed for the immobilization of Schiff base complexes on NFS materials and represented as NFS-[Co(salen) $]^{+}$, NFS-[Co(salophen) $]^{+}$, NFS-[Mn(salen) $]^{+}$and NFS-[Mn(salophen) $]^{+}$.

\subsection{Fabrication of electrodes}

An aqueous colloidal solution $(0.5-1.0 \%)$ of the respective material was prepared together with $0.01 \%$ poly (vinyl alcohol). Using alumina slurry and cloth pad, glassy carbon electrodes (GCE, $0.07 \mathrm{~cm}^{2}, \mathrm{CH}$ Instruments) were polished to mirror finish. The polished GCE was washed and subjected to sonication with water and methanol. The colloidal solution was dropped (typically $5 \mu \mathrm{L}$ ) on the vertically mounted polished dry GCE and dried in air for about $12 \mathrm{~h}$. These electrodes are represented as GCE/respective material. After complete drying, the electrode was used for electrochemical studies.

\subsection{Instrumentation}

For X-ray diffraction (XRD) measurements ( $2 \theta$ value from $10^{\circ}$ to $80^{\circ}$ ), 1D 3000 SEIFERT diffractometer and $\mathrm{Cu}-\mathrm{K} \alpha$ radiation were used. UV-Vis spectrophotometers (Shimadzu, UV 1700 Pharma Spec, and UNICO, 2802 PC) were used to measure the absorption spectra of the synthesized materials. To record the absorption spectra of the powdered silica materials, they were mixed intimately with few drops of Nujol and the resulting mull was applied on a suitably cut Whatman filter paper. The mull applied filter paper was placed on the path of the light to measure the absorbance. Transmission electron microscope (TEM) images of the silica materials were collected using TECNAI $20 \mathrm{G}^{2}$ FEI microscope and it was operated at $200 \mathrm{KV}$. CHI660C (CH Instruments) electrochemical workstation was used for cyclic voltammetry analysis at room temperature $\left(25 \pm 2^{\circ} \mathrm{C}\right)$. The silica materials modified GCEs as working electrodes, aqueous $\mathrm{Ag} / \mathrm{AgCl}$ as reference electrode and a thin platinum wire as counter electrode were used for the electrochemical studies.

\section{Results and Discussion}

\subsection{Materials characterization}

TFS and NFS materials with and without M NPs and/or adsorbed catalysts were first analysed by absorption 
spectra. As shown in figure 1A, TFS material without M NPs and catalyst, exhibit no absorption peaks in the visible region. ${ }^{2,11,12,18,20}$ Surface plasmon resonance bands of corresponding M NPs are observed in the $\mathrm{Ag}$ and $\mathrm{Au}$ NPs containing silica materials at 464 and $551 \mathrm{~nm}$ (Ag-TFS and Au-TFS), respectively. ${ }^{2,11,12,18,20,44}$ TFS, Ag-TFS and Au-TFS adsorbed with MPcs (TFS-ZnPc, Ag-TFS-ZnPc, AuTFS-ZnPc, TFS-CoPc and Au-TFS-CoPc) show their corresponding absorption bands around $610-620 \mathrm{~nm}$ (for $\mathrm{CoPc}$ ) and 670-680 nm (for $\mathrm{ZnPc}$ ). Since these absorption spectra are comparable with the spectra of corresponding MPcs in DMF solution (Fig. not shown), the adsorption of MPcs on the silica materials is confirmed. . $^{211,18,20}$ The absorbance spectra of NFS-[Co(salen) $]^{+}$, NFS-[Co(salophen) $]^{+}$, NFS$[\mathrm{Mn}(\text { salen })]^{+}$and NFS-[Mn(salophen $\left.)\right]^{+}$materials ${ }^{19,40}$ are shown in figure 1B. Resemblance of these spectra to their corresponding solution spectra evidences
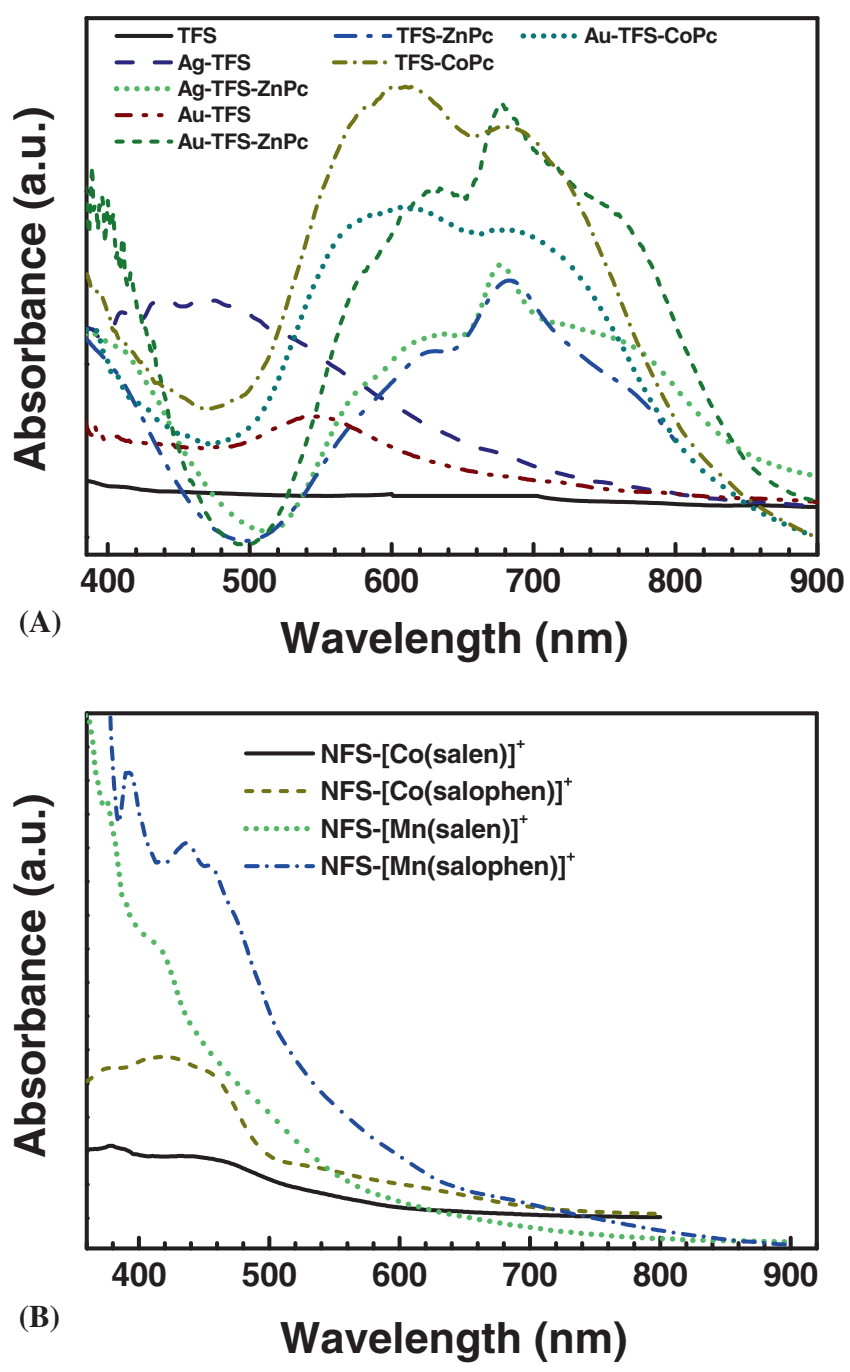

Figure 1. UV-vis absorption spectra of TFS (A) and NFS (B) materials. the incorporation/adsorption of Schiff base metal complexes into the NFS. XRD patterns of the materials can provide knowledge on crystallinity of the synthesized material as well as the incorporation of M NPs. Therefore, XRD patterns are recorded in the range where $M$ NPs show their reflections (figure 2). TFS does not exhibit any peak in the $2 \theta$ range of $10^{\circ}$ to $80^{\circ}$. M NPs incorporated silica materials show four peaks around $38.1^{\circ}, 44.2^{\circ}, 64.4^{\circ}$, and $77.4^{\circ}$ corresponding to their cubic structure. MPcs adsorbed Ag-TFS and Au-TFS show several additional peaks in the $2 \theta$ range of $10^{\circ}$ to $30^{\circ}$, which represent the crystallinity of the adsorbed $\mathrm{ZnPc}$ or CoPc. The shape and size of TFS materials are not changed by further incorporation of M NPs and/or MPcs. ${ }^{2,11,18,20,25}$ Incorporation and uniform distribution of M NPs on silica materials is revealed by the images of Ag-TFS and Au-TFS materials. ${ }^{2,11,18,20,25}$ Adsorption of MPcs on TFS and M-TFS materials slightly changes the morphology of the parent material which confirms the adsorption of MPcs on silica materials. ${ }^{2,11,18,20,25}$

\subsection{Electrochemical characterization}

Cyclic voltammetry (CV) was used to evaluate the characteristics of a catalyst immobilized in a matrix. The NFS and TFS material did not show any redox process (Figure not shown) in the region from -0.5 to 1.5 V. The Ag and Au NPs incorporated TFS materials show distinct redox processes due to the oxidation/reduction of respective M NPs (figure 3). Ag-TFS coated GCE (GCE/Ag-TFS) exhibits stable redox process which may be assigned to oxidation $\left(\mathrm{Ag}^{0} \mathrm{Ag}^{+}\right.$at

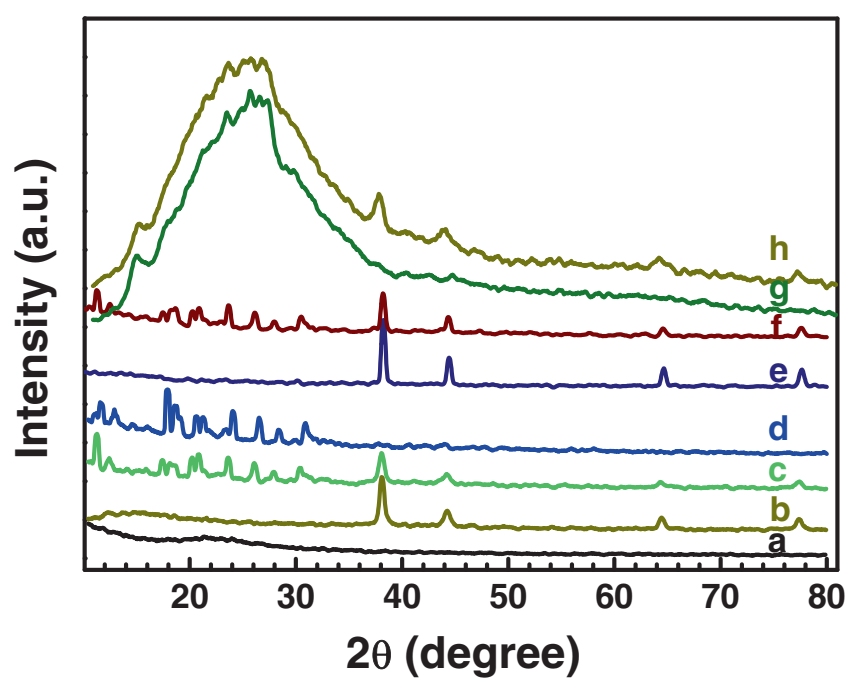

Figure 2. XRD reflection patterns of TFS (a), Ag-TFS (b), Ag-TFS-ZnPc (c), TFS-ZnPc (d), Au-TFS (e), Au-TFS$\mathrm{ZnPc}$ (f) TFS-CoPc (g) and Au-TFS-CoPc (h) materials. 


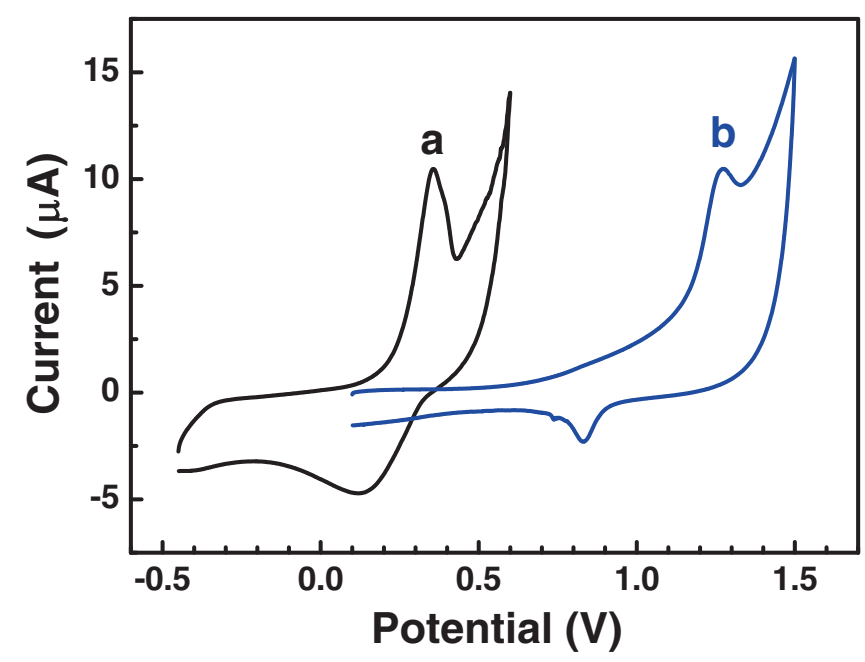

Figure 3. Cyclic voltammograms of (a). GCE/Ag-TFS and (b). GCE/Au-TFS in $0.05 \mathrm{M} \mathrm{HClO}_{4}$. Scan rate: $50 \mathrm{mVs}^{-1}$.

$359 \mathrm{mV})$ and reduction $\left(\mathrm{Ag}^{+} \mathrm{Ag}^{0}\right.$ at $\left.135 \mathrm{mV}\right)$ reactions of Ag NPs. Au-TFS also exhibits (GC/Au-TFS) similar redox process and it is assigned to the formation (at $1275 \mathrm{mV}$ ) and reduction $(836 \mathrm{mV}$ ) of gold oxide. MPcs adsorbed TFS materials (with and without M NPs) are characterized by the presence of distinct redox peaks (figure 4) due to $\mathrm{CoPc}$ or ZnPc. All the synthesized materials display stable and reproducible response when coated on GCE. The CV response of TFS-ZnPc, TFS-CoPc, Ag-TFS-ZnPc, Au-TFS-ZnPc and Au-TFSCoPc materials coated GCE (represented as GCE/TFSZnPc, GCE/TFS-CoPc, GCE/Ag-TFS-ZnPc, GCE/AuTFS-ZnPc and GCE/Au-TFS-CoPc, respectively) are

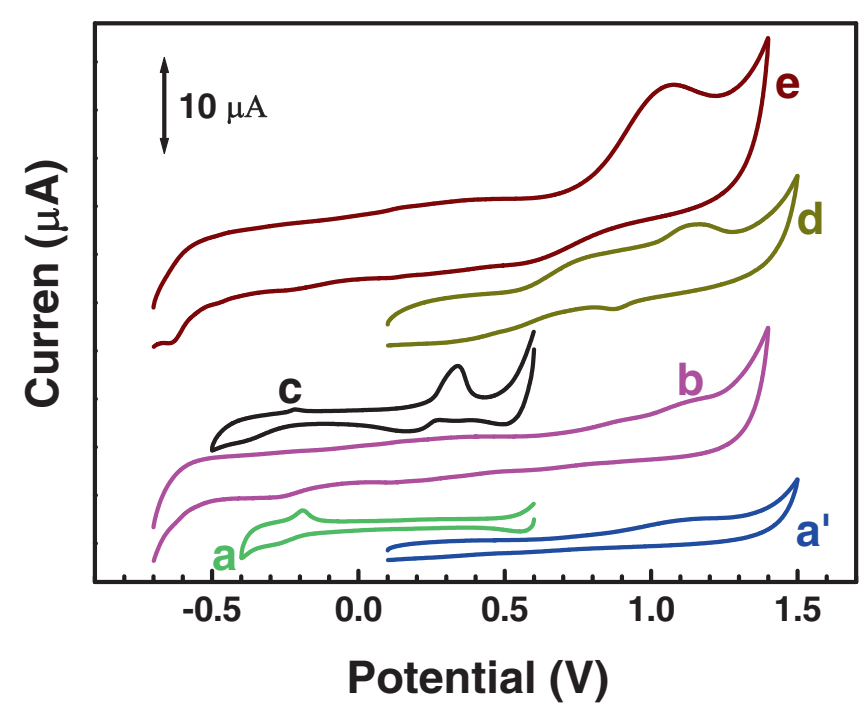

Figure 4. Cyclic voltammograms of GCE/TFS-ZnPc (a, a/), GCE/TFS-CoPc (b), GCE/Ag-TFS-ZnPc (c), GCE/ $\mathrm{Au}-\mathrm{TFS}-\mathrm{ZnPc}$ (d) and GCE/Au-TFS-CoPc (e) in $0.05 \mathrm{M}$ $\mathrm{HClO}_{4}$. Scan rate: $50 \mathrm{mVs}^{-1}$. shown in figure 4. In the absence of M NPs, i.e., TFS$\mathrm{ZnPc}$ and TFS-CoPc materials reveal redox response due to the corresponding MPc (curves $a, a^{\prime}$ and $b$ of figure 4). A pair of redox peaks due to phthalocyanine (Pc) ligand reduction and oxidation $\left(\mathrm{Zn}^{\mathrm{II}} \mathrm{Pc}(-2) / \mathrm{Zn}{ }^{\mathrm{II}} \mathrm{Pc}(-\right.$ $3)^{-}$) appears (figure $\left.4 a\right)^{2,45}$ with an $E_{1 / 2}$ value of -230 $\mathrm{mV}$ (scan rate: $50 \mathrm{mVs}^{-1}$ ) at GCE/TFS-ZnPc. In addition, a pair of broad redox peaks (not well defined, figure $\left.4 \mathrm{a}^{\prime}\right)$ attributed to the oxidation and reduction of Pc ligand $\left(\mathrm{Zn}^{\mathrm{II}} \mathrm{Pc}(-2)\right.$ to $\left.\mathrm{Zn}{ }^{\mathrm{II}} \mathrm{Pc}(-1)^{+}\right)$is observed. ${ }^{45}$ Further, an irreversible peak due to the Pc ligand oxidation is detected in the high positive potential region, (around $1.120 \mathrm{~V}$ ) for the adsorbed $\mathrm{ZnPc}$ (figure $4 \mathrm{a}^{\prime}$ ). Similarly, two weak redox couples are observed for TFS-CoPc material (figure $4 \mathrm{~b}$ ) with $E_{1 / 2}$ values around 0.12 and $0.34 \mathrm{~V}$. Even though at low scan rates $\left(20 \mathrm{mVs}^{-1}\right.$ or below), the redox couples are realized a bit better, for comparison purpose, the $\mathrm{CV}$ response at $50 \mathrm{mVs}^{-1}$ is shown (figure $4 \mathrm{~b}$ ). In addition, a quasireversible Pc ligand reduction with a peak potential at $-260 \mathrm{mV}$ due to the formation of $\mathrm{Co}^{\mathrm{I}} \mathrm{Pc}(-3)^{2-}$ and an irreversible Pc ligand oxidation at high positive potential $(1127 \mathrm{mV})$ due to the formation of $\mathrm{Co}^{\mathrm{III}} \mathrm{Pc}(-1)$ are also observed. When M NPs are introduced into the materials, they exhibit electrochemical properties of the respective M NPs in addition to the adsorbed MPc. GCE/Ag-TFS-ZnPc shows a small shift in both $\mathrm{Ag}^{0 /+}$ redox couple and $\mathrm{Zn}^{\text {II }} \mathrm{Pc}(-2) / \mathrm{Zn}^{\mathrm{II}} \mathrm{Pc}(-3)^{-}$redox process (figure $4 \mathrm{c}$ ). These shifts could be due to the interaction between the Ag NPs and ZnPc. Likewise, GCE/ $\mathrm{Au}-\mathrm{TFS}-\mathrm{ZnPc}$ (figure 4d) and GCE/Au-TFS-CoPc (figure 4e) also exhibit similar trend.

$\mathrm{CV}$ response of GCE/NFS-[Co(salen) $]^{+}$(figure 5a) and GCE/NFS-[Co(salophen) $]^{+}$(figure $5 b$ ) electrodes exhibit stable redox peaks which are attributed to $[\mathrm{Co}$ (salen $)]^{+}$to $[\mathrm{Co}($ salen $)]$ and $[\mathrm{Co}$ (salophen $\left.)\right]^{+}$to $[\mathrm{Co}($ salophen $)]$ redox processes, respectively. ${ }^{19,46,47}$ The calculated $E_{1 / 2}$ values for the redox process are 423 and $378 \mathrm{mV}$, respectively (scan rate: $150 \mathrm{mV}$ ). Similarly, NFS-[Mn(salen) $]^{+}$(figure 5c) and NFS$\left[\mathrm{Mn}(\text { salophen) }]^{+}\right.$(figure $5 \mathrm{~d}$ ) materials display $E_{1 / 2}$ values corresponding to their $[\mathrm{Mn}(\text { salen })]^{+}$to $[\mathrm{Mn}($ salen$)]$ and $[\mathrm{Mn}(\text { salophen })]^{+}$to $[\mathrm{Mn}($ salophen $)]$ redox process at 303 and $706 \mathrm{mV}$, respectively. ${ }^{40}$

\subsection{Electrocatalytic properties}

It is well-known that oxygen and carbon dioxide can be electrocatalytically reduced by MPs and MPcs however, with less efficiency. ${ }^{32-35}$ Suitable immobilization of the electrocatalyst on the electrode surface could increase the efficiency of the electrocatalytic process due to the 


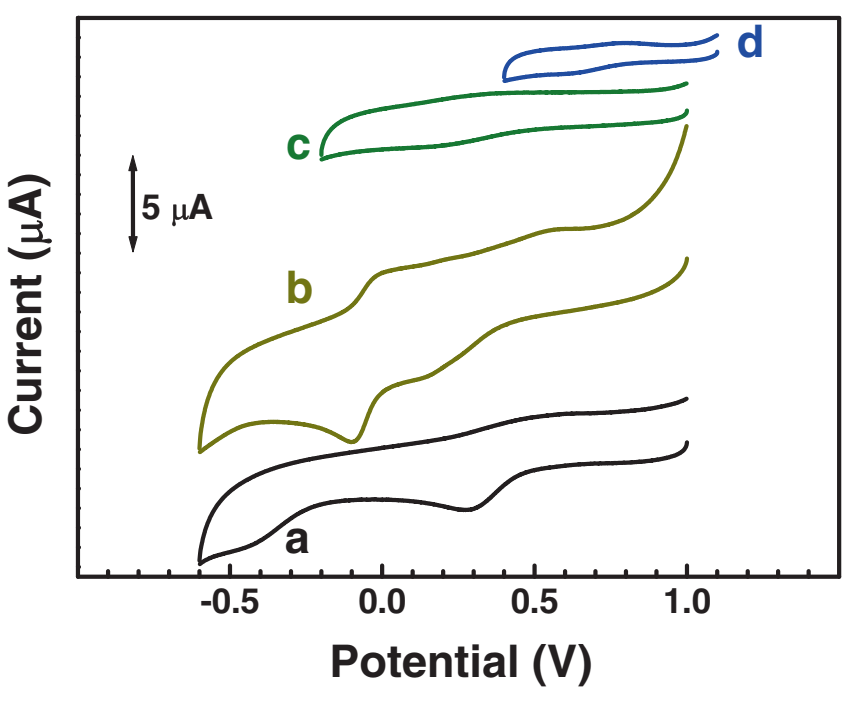

Figure 5. Cyclic voltammograms of GCE/NFS-[Co $($ salen $)]^{+}$(a), GCE/NFS- $[\mathrm{Co}(\text { salophen })]^{+}(\mathbf{b})$, GCE/NFS$[\mathrm{Mn}(\text { salen })]^{+}$and GCE/NFS-[Mn(salophen $\left.)\right]^{+}$in $0.05 \mathrm{M}$ $\mathrm{HClO}_{4}$. Scan rate: $150 \mathrm{mVs}^{-1}$.

preconcentration of catalyst molecules in a small area, and large fraction of electrochemically active catalyst. Therefore, M NPs and/or CoPc/ZnPc are immobilized on the electrode surface as electrocatalysts. To analyse the ability of these new materials towards electrocatalytic reduction of oxygen, cyclic voltammograms are recorded in $0.05 \mathrm{M} \mathrm{HClO}_{4}$ acidic medium and shown in figure 6 (for clarity, reductive scans alone shown). GCE/Ag-TFS in the presence of oxygen saturated $0.05 \mathrm{M} \mathrm{HClO}_{4}$ solution shows a distinct peak for the reduction of oxygen around $-243 \mathrm{mV}$. Under similar conditions, GCE/Au-TFS also exhibits reduction of

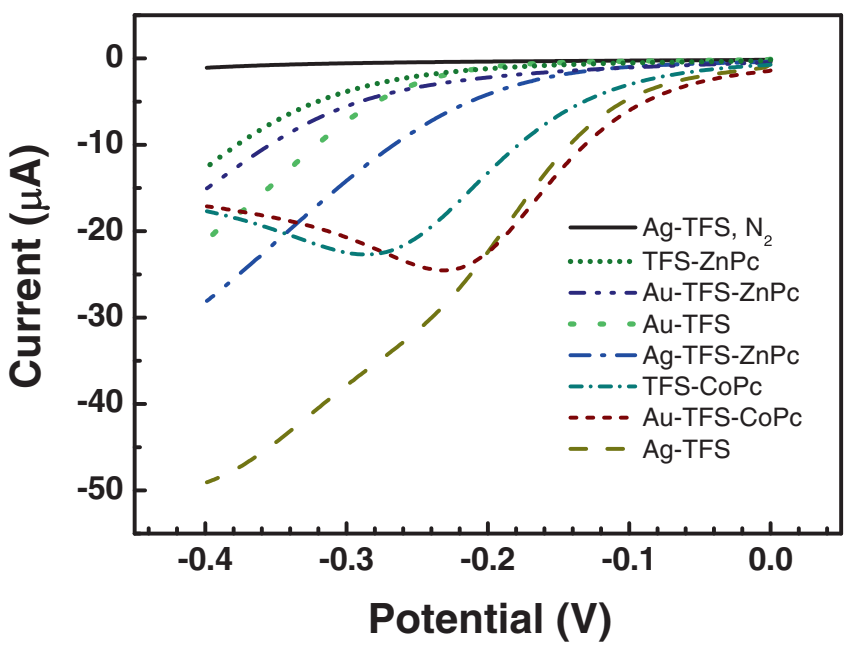

Figure 6. Electrocatalytic reduction of oxygen at TFS material coated GCEs in $0.05 \mathrm{M} \mathrm{HClO}_{4}$. The solid line indicates the reduction scan of GCE/Ag-TFS under the nitrogen saturated condition. Scan rate: $50 \mathrm{mVs}^{-1}$. oxygen, however without a defined peak. Similarly all the other materials show electrocatalytic oxygen reduction except the TFS which contains no electrocatalyst (either M NPs or MPc). The high reduction current observed at GCE/Ag-TFS could be due to the large amount of Ag NPs incorporated in the TFS matrix. It should also be noted that the size and shape of the NPs is another important factor in determining the catalytic process. When $\mathrm{CoPc}$ or $\mathrm{ZnPc}$ is introduced further to the Ag/Au-TFS material, the electrocatalytic current is supposed to increase because of the synergetic effect between MPc and MNPs. As expected, MNPs and MPc synergize towards the electrocatalytic oxygen reduction as evidenced from the increase in current in figure 6. M NPs act as tiny electron conduction centres or nanoelectrodes and increase the electrical communication within the film, thereby facilitating the electron transfer. ${ }^{12}$

Oxygen electroreduction at NFS and Schiff base metal complex adsorbed NFS materials are also analysed $^{19,48}$ It is found that the Schiff base metal complex adsorbed NFS materials show efficient electrocatalytic activity towards the electroreduction of oxygen (figure 7). In oxygen saturated solutions, cyclic voltammograms (for clarity, only the reduction cycles are shown) of GCE/NFS$[\mathrm{Co}(\text { salen })]^{+}$, GCE/NFS-[Co(salophen) $]^{+}$, GCE/TFS$[\mathrm{Mn}(\text { salen })]^{+}$and GCE/TFS-[Mn(salophen) $]^{+}$show increased reduction current in comparison to the nitrogen saturated solutions. Even though no defined peaks are observed, the enormous increase in reduction current in presence of oxygen clearly indicates the electrocatalytic reduction of oxygen at these electrodes. As observed, at NFS material, inefficient reduction of oxygen starts at around $-230 \mathrm{mV}$, whereas in all Schiff

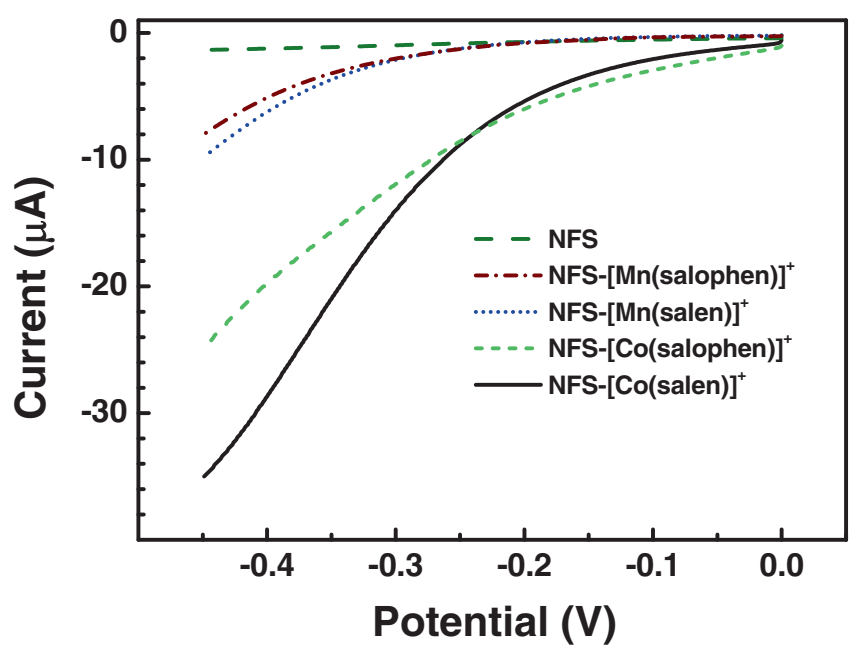

Figure 7. Electrocatalytic reduction of oxygen at NFS material coated GCEs in $0.05 \mathrm{M} \mathrm{HClO}_{4}$ Scan rate: $50 \mathrm{mVs}^{-1}$. 
base complex adsorbed materials, the oxygen reduction is observed at low negative potentials.

The TFS materials are further analysed for the electrocatalytic reduction of $\mathrm{CO}_{2}{ }^{2,34,49}$ Since the catalytic efficiency is expected to increase for immobilized catalysts, $\mathrm{CO}_{2}$ reduction is studied using these new materials (figure 8). Electrocatalytic reduction of $\mathrm{CO}_{2}$ is observed only in the presence of $\mathrm{ZnPc} .^{2} \mathrm{TFS}$, Ag-TFS and Au-TFS did not show any electrocatalytic properties towards $\mathrm{CO}_{2}$ reduction. TFS-ZnPc (figure 8a), Ag-TFS-ZnPc (figure 8b) and Au-TFS$\mathrm{ZnPc}$ (figure $8 \mathrm{c}$ ) materials show electrocatalytic reduction of $\mathrm{CO}_{2}$ around $-0.50 \mathrm{~V}$. Earlier investigations ${ }^{50}$ on the $\mathrm{CO}_{2}$ electroreduction indicate that it occurs at high negative potentials (around $-1.00 \mathrm{~V}$ ) even in the presence of electrocatalysts such as $\mathrm{Ru}\left(2,2^{\prime}-\text { bithiazole }\right)_{3}^{2+}$. Thus, a large shift in the $\mathrm{CO}_{2}$ electroreduction (more than $500 \mathrm{mV}$ ) occurs in the presence of $\mathrm{ZnPc}$ (figure 8a). Thus, the efficiency of the $\mathrm{ZnPc}$ is greatly increased in the presence of Au NPs, which is evidenced by the large increase in the $\mathrm{CO}_{2}$ electroreduction current (figure $8 \mathrm{~b}$ and $\mathrm{c}$ ).

Since MPcs are known to exhibit several catalytic reactions, the $\mathrm{ZnPc}$-adsorbed silica materials are tested for nitrite reduction in acidic medium. ${ }^{11}$ In the absence and presence of nitrite, GCE/TFS electrode does not show any faradaic process indicating that nitrite is not reduced at this electrode. At GCE/TFS-ZnPc, nitrite reduction occurs less efficiently after $-30 \mathrm{mV}$. However, at GCE/Ag-TFS and GCE/Ag-TFS-ZnPc

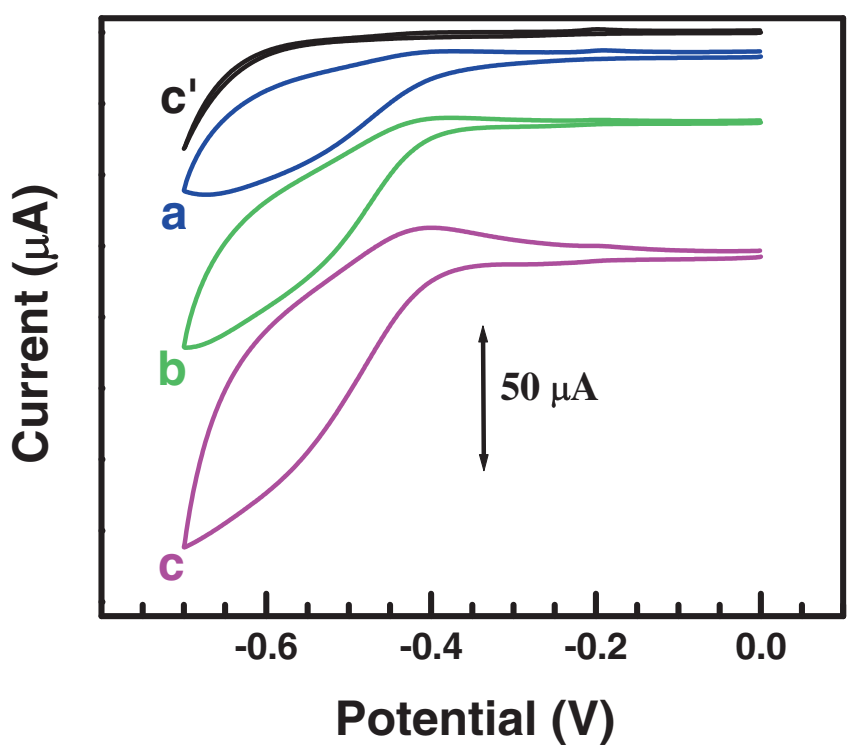

Figure 8. Cyclic voltammograms showing the electrocatalytic reduction of $\mathrm{CO}_{2}$ at the TFS-ZnPc (a), Ag-TFS-ZnPc (b) and Au-TFS-ZnPc (c) material coated GCEs. Curve c' is recorded at GCE/Au-TFS-ZnPc under nitrogen saturated condition. Scan rate: $50 \mathrm{mVs}^{-1}$.

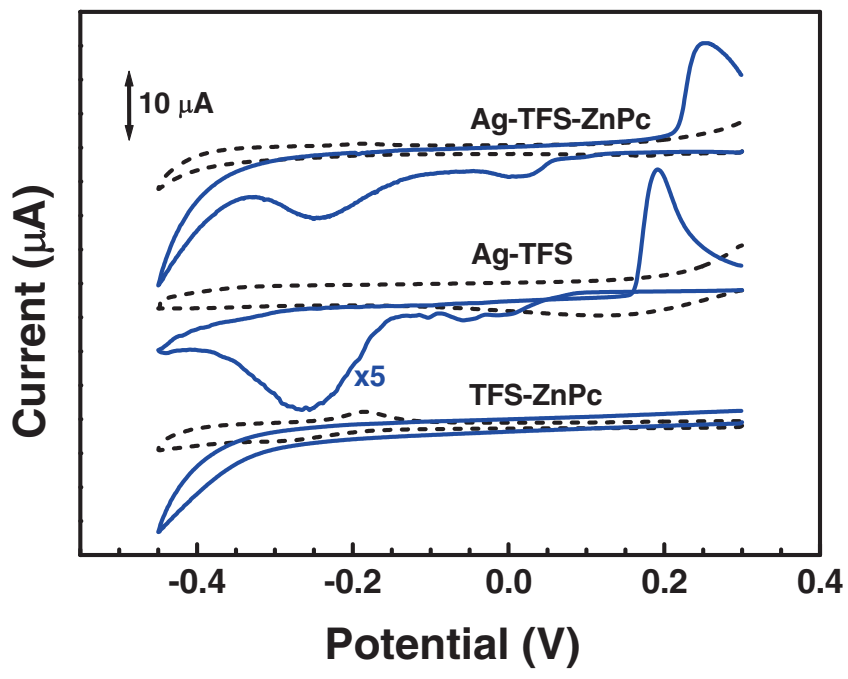

Figure 9. Cyclic voltammograms showing the electrocatalytic reduction of nitrite $(20.0 \mathrm{mM})$ at the TFS-ZnPc, AgTFS and Ag-TFS-ZnPc material coated GCEs. The dashed lines indicate the cyclic voltammograms of the corresponding GCEs in the absence of nitrite. Scan rate: $50 \mathrm{mVs}^{-1}$.

electrodes, nitrite is electrocatalytically reduced at -0.01 and $0.02 \mathrm{~V}$, respectively (figure 9). The electrocatalysis shown by $\mathrm{ZnPc}$ (i.e., at GC/TFS-ZnPc) towards the electroreduction of nitrite is influenced in the presence of Ag NPs (i.e., at GC/Ag-TFS-ZnPc) as evidenced by 12 times increase in the reduction current at $-0.25 \mathrm{~V}$ (figure 9a). The large increase in current at GC/Ag-TFS compared with GC/Ag-TFS-ZnPc could be due to the high amount of Ag NPs in the material.

\section{Summary}

The use of MCM-41 type materials, TFS and NFS in electrocatalysis and their possible application as fuel cell material are demonstrated. Metal complexes and/or metal NPs are firmly immobilized into/onto the TFS and NFS materials. They act as durable electrode modifiers with strong adhering property. Presence of metal NPs greatly improves the electrocatalytic reactions such as oxygen, carbon dioxide and nitrite reductions.

\section{Acknowledgements}

The author thanks UGC, CSIR and DST, New Delhi for funding. The author also thanks Prof. S K Sengupta and Dr. Manas Pal for useful suggestions.

\section{References}

1. Walcarius A and Kuhn A 2008 Trends Anal. Chem. 27 593 
2. Pal M and Ganesan V 2009 Langmuir 2513264

3. Walcarius A and Delacote C 2003 Chem. Mater. 154181

4. Ganesan V and Walcarius A 2004 Langmuir 203632

5. Walcarius A and Ganesan V 2006 Langmuir 22469

6. Kresge C T, Leonowicz M E, Roth W J, Vartuli J C and Beck J S 1992 Nature 359710

7. Beck J S, Vartuli J C, Roth W J, Leonowicz M E, Kresge C T, Schmitt K D, Chu C T W, Olson D H, Sheppard E W, McCullen S B, Higgins J B and Schlenker J L 1992 J. Am. Chem. Soc. 11410834

8. Walcarius A, Etienne M and Bessiere J 2002 Chem. Mater. 142757

9. Walcarius A, Etienne M and Lebeau B 2003 Chem. Mater. 152161

10. Walcarius A 2013 Chem. Soc. Rev. 424098

11. Pal M and Ganesan V 2010 Electrochim. Acta 554071

12. Pal M and Ganesan V 2010 Analyst 1352711

13. Jena B K and Raj C R 2007 J. Phys. Chem. C 1116228

14. Ramendra D S, Raj B K and Raj R C 2013 Anal. Bioanal. Chem. 4053431

15. Vasimalai N and John A S 2014 Sens. Actuators B 190 800

16. Azad U P and Ganesan V 2010 Chem. Commun. 466156

17. Azad U P, Ganesan V and Pal M 2011 J. Nanopart Res. 133951

18. Pal M and Ganesan V 2012 J. Electroanal. Chem. 6727

19. Pal M and Ganesan V 2012 Catal. Sci. Tech. 22383

20. Pal M, Ganesan V and Azad U P 2012 Thin Solid Films 525172

21. Rastogi P K, Ganesan V and Krishnamoorthi S 2014 J. Mater. Chem. A 2933

22. Rastogi P K, Ganesan V and Krishnamoorthi S 2014 Electrochim. Acta 1148

23. Boyen H G, Herzog T, Kastle G, Weigl F, Ziemann P, Spatz J P, Moller M, Wahrenberg R, Garnier M G and Oelhafen P 2002 Phys. Rev. B 6575412

24. Akolekar D B and Bhargava S K 2005 J. Mol. Catal. A Chem. 23677

25. Etienne M, Lebeau B and Walcarius A 2002 New J. Chem. 26384

26. Martin C R, Rubinstein I and Bard A J 1982 J. Am. Chem. Soc. 1044817

27. Buttry D A and Anson F C 1983 J. Am. Chem. Soc. 105 685

28. White H S, Leddy J and Bard A J 1982 J. Am. Chem. Soc. 1044811
29. Ramaraj R 1996 Proc. Indian Acad. Sci. Chem. Sci. 108 181

30. Ernst S and Selle M 1999 Micro. Meso. Mat. 27355

31. Lu X B, Wang H and He R 2002 J. Mol. Catal. A Chem. 18633

32. Fuerte A, Corma A, Iglesias M, Morales E and Sanchez F 2006 J. Mol. Catal. A Chem. 246109

33. Ponce I, Silva J F, Onate R, Rezende M C, Paez M A, Zagal J H and Pavez J 2012 J. Phys. Chem. C, 116, 15329

34. Magdesieva T V, Yamamoto T, Tryk T A and Fujishima A 2002 J. Electrochem. Soc. 149 D89

35. Sonoyama N, Kirii M and Sakata T 1999 Electrochem. Commun. 1213

36. Chebotareva N and Nyokong T 1997 J. Appl. Elechem. 27975

37. Silva S D, Shan D and Cosnier S 2004 Sens. Actuators B 103397

38. Narvaez A, Dominguez E, Katakis I, Katz E, Ranjit K T, BenDov I and Willner I 1997 J. Electroanal. Chem. 430 227

39. Hwang S, Lee J and Kwak J 2005 J. Electroanal. Chem. 579143

40. Ganesan V, Pal M and Tiwari M 2014 Bull. Mater. Sci. 37623

41. Bottcher A, Elian H, Jager E G, Langfelderova H, Mazur M, Muller L, Paulus H, Pelikan P, Rudolph, M and Valko M 1993 Inorg. Chem. 324131

42. Chen H, Cronin J A, and Archer R D 1995 Inorg. Chem. 342306

43. Kantam M L and Bharathi B 1998 Catal. Lett. 55235

44. Rodrigues S, Uma S, Martyanov I N and Klabunde K J 2005 J. Mol. Catal. A Chem. 233405

45. Giraudeau A, Louati A, Gross M, Andre J, Simon J, Su C H and Kadish K M 1983 J. Am. Chem. Soc. 105 2917

46. Okada T, Gotou S, Yoshida M, Yuasa M, Hirose T and Sekine I 1999 J. Inorg. Organomet. Polym. 9, 199

47. Moad A J, Klein L J, Peters D G, Karty J A and Reilly J P 2002 J. Electroanal. Chem. 531163

48. Geigar T and Anson F C 1981 J. Am. Chem. Soc. 103 7489

49. Eggins B R, Irvine J T S and Grimshaw J 1989 J. Electroanal. Chem. 266125

50. Begum A and Pickup P G 2007 Electrochem. Commun. 92525 